\title{
The Central Spike Complex of Bacteriophage T4 Contacts PpiD in the Periplasm of Escherichia coli
}

\author{
Sabrina Wenzel ${ }^{1}$, Mikhail M. Shneider ${ }^{2}$, Petr G. Leiman $\left.{ }^{3}{ }^{(}\right)$, Andreas Kuhn ${ }^{1, *(\mathbb{D})}$ and \\ Dorothee Kiefer $^{1}$ \\ 1 Institute of Biology, University of Hohenheim, 190h, Garbenstr. 30, 70599 Stuttgart, Germany; \\ Sabrina.Wenzel@uni-hohenheim.de (S.W.); Dorothee.Kiefer@uni-hohenheim.de (D.K.) \\ 2 Shemyakin-Ovchinnikov Institute of Bioorganic Chemistry, Laboratory of Molecular Bioengineering, \\ 16/10 Miklukho-Maklaya St., 117997 Moscow, Russia; mm_shn@mail.ru \\ 3 Department of BMB, Basic Sciences Building 6.600D, University of Texas Medical Branch, \\ 301 University Blvd, Galveston, TX 77555-0647, USA; pgleiman@utmb.edu \\ * Correspondence: Andreas.Kuhn@uni-hohenheim.de; Tel.: +49-(0)711-45922222; Fax: +49-(0)711-45922238
}

Received: 4 September 2020; Accepted: 2 October 2020; Published: 6 October 2020

\begin{abstract}
Infecting bacteriophage T4 uses a contractile tail structure to breach the envelope of the Escherichia coli host cell. During contraction, the tail tube headed with the "central spike complex" is thought to mechanically puncture the outer membrane. We show here that a purified tip fragment of the central spike complex interacts with periplasmic chaperone PpiD, which is anchored to the cytoplasmic membrane. PpiD may be involved in the penetration of the inner membrane by the T4 injection machinery, resulting in a DNA-conducting channel to translocate the phage DNA into the interior of the cell. Host cells with the ppiD gene deleted showed partial reduction in the plating efficiency of T4, suggesting a supporting role of PpiD to improve the efficiency of the infection process.
\end{abstract}

Keywords: bacteriophage T4; tail structure; periplasmic chaperone; DNA translocation

\section{Introduction}

The adsorption and penetration of myophage T4 is a fascinating example of a self-triggered multistep event in molecular biology. The process is divided into five steps: (1) the adsorption of long tail fibres; (2) the binding of short tail fibres; (3) tail-sheath contraction; (4) tail-tube movement through the outer membrane and periplasm; and (5) the contact of the tail-tube tip with the inner membrane to initiate the phage DNA ejection process.

Bacteriophage T4 contacts its host cells by initially stochastic low-affinity binding six long tail fibres to the outer membrane [1-3]. This binding triggers a conformational change in the geometry of the baseplate that results in stretching out six short tail fibres (gp12), leading to an irreversible and firm binding of the phage to the bacterial lipopolysaccharide moiety of the cell $[4,5]$. The conformational transition of the baseplate to a starlike configuration then starts the contraction of the tail structure [6]. Thereby, the gp18 proteins of the tail sheath rearrange their intersubunit contacts, leading to wavelike shortening of the sheath $[7,8]$. Consequently, the tail sheath is shortened from 92.5 to $42 \mathrm{~nm}$, whereas the inner tail tube stays in its stiff cylinder-like structure that is mechanically forced to move through the centre of the baseplate.

The movement of the tail tube towards the cell surface takes baseplate components gp5.4, gp5, gp27, gp48, and gp54 along at the leading end of the tube (gp19); gp5.4, at the tip of this structure, is tightly connected to the C-terminal part of gp5, which forms a stable triple helix, and is called the gp5C-gp5.4 central spike complex (Figure 1A) [9,10]. 
A

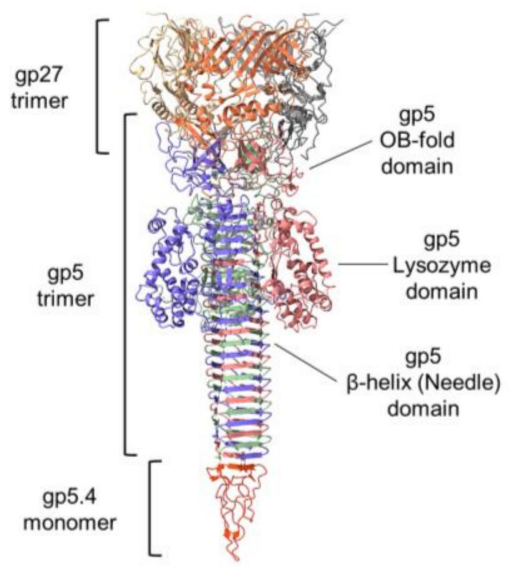

B

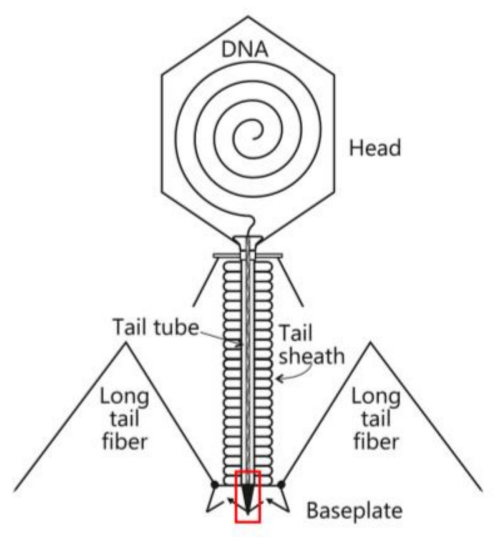

cleavage

C

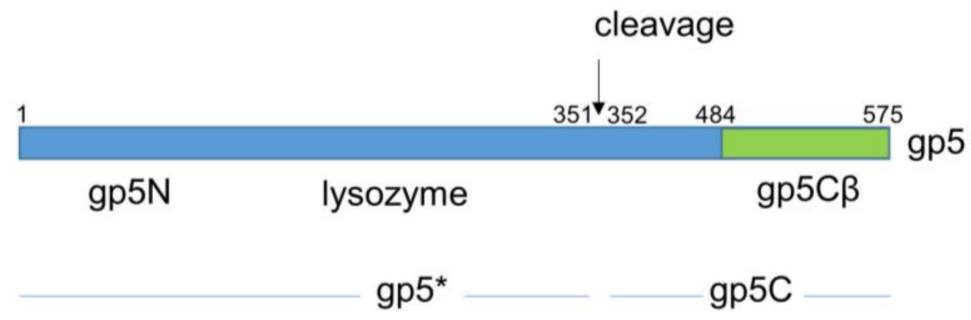

Figure 1. (A) Ribbon diagram of T4 central spike complex extracted from T4 baseplate structure [5]. Each chain shown in distinct colour. The gp27 hub protein forms an extension to the tail tube. It holds the N-terminal oligosaccharide/oligonucleotide binding fold (OB-fold) domain of the gp5 spike protein in its central channel. The gp5 spike also contains a middle lysozyme domain and a C-terminal $\beta$ helix domain. The blunt tip of the $\beta$ helix is sharpened by spike tip gp5.4. (B) Schematic structure of the T4 phage particle [8]. Location of the spike complex shown as a red rectangle. (C) Schematic of the gene 5 derived products. The gp5 protein is cleaved at position 351 after its assembly into the phage, resulting in gp5* and gp5C [11]. The carboxyl-terminal part of gp5C (green) encompassing the triple $\beta$ helix that starts at residue 484 was cloned onto a plasmid [10].

Gp5 exists in the phage as a cleaved protein, with the N-terminal part (gp5*) bound to gp27 and the C-terminal part tightly bound to gp5.4. The penetration of the outer membrane is most likely executed by mechanical force generated by the sheath contraction and applied to the central spike structure. In the periplasm, the tip of the tail moves through the peptidoglycan layer, which is facilitated by the mureolytic activity of the lysozyme domain of gp5.

The molecular details that lead to contact with the inner membrane and to the translocation of the phage DNA through the membrane into the cytoplasm are still unknown. Here, a number of scenarios are discussed. The needle at the tip of the tail tube may come into contact with the inner membrane and initiate an opening of a DNA-conductive channel. Second, the central spike complex dissociates from the tail tube in the periplasm, leaving the N-terminal part of gp5 exposed on the tube tip, which could then come into contact with the inner membrane. Furthermore, it is possible that, in the periplasm, the entire T4 tip structure dissociates from the tube, and gp27 or gp 48 comes into contact with the inner membrane for DNA translocation.

To shed some light on these interesting events, we purified the gp5C $\beta$-gp5.4 complex, a tip fragment of the central spike complex, and tested its binding to liposomes and spheroplasts. We found that this spike fragment binds to periplasmic protein PpiD that is anchored to the inner membrane. PpiD is a periplasmic chaperone [12] that is connected to the SecYEG translocon [13]. A chromosomal deletion of PpiD resulted in a decrease of T4 plating efficiency on this strain, suggesting that PpiD might play an important role in $\mathrm{T} 4$ infection. 


\section{Materials and Methods}

\subsection{Bacterial Strains and Phage}

T4 was propagated on E. coli B and on BW25113 (parental strain of $\Delta p p i D$; Keio collection) in Luria broth (LB medium). Media preparation and bacterial growth were performed according to standard methods [14]. ppiD deletion strain JW0431 was cultured in LB medium containing $25 \mu \mathrm{g} / \mathrm{mL}$ kanamycin at $37^{\circ} \mathrm{C}$. E. coli BL21 (DE3) [15], transformed with plasmid pEEva2 encoding the gp5C $\beta-5.4$ complex or plasmid pASK-IBA3C encoding PpiD, respectively, was grown in LB in the presence of ampicillin $(100 \mu \mathrm{g} / \mathrm{mL}$, final concentration).

\subsection{Purification of $g p 5 C \beta-g p 5.4$ Complex}

E. coli BL21 (DE) harbouring plasmid pEEva2 encoding the His-gp5C $\beta$-gp5.4 complex was grown in a 2xYT medium containing $100 \mu \mathrm{g} / \mathrm{mL}$ ampicillin at $37^{\circ} \mathrm{C}$ until the culture had reached $\mathrm{OD}_{600}$ of 0.6 . The culture was cooled down to $18^{\circ} \mathrm{C}$. Gp5C $\beta-5.4$ complex expression was induced by the addition of isopropyl- $\beta$-D-thiogalactopyranoside (IPTG) to $0.5 \mathrm{mM}$, and the culture was grown at $18{ }^{\circ} \mathrm{C}$ overnight. Cells were harvested by centrifugation at $8000 \times g$ at $4{ }^{\circ} \mathrm{C}$ for $15 \mathrm{~min}$. Cell pellets were resuspended in a lysis buffer (20 mM Tris- $\mathrm{HCl}(\mathrm{pH}$ 8), $300 \mathrm{mM} \mathrm{NaCl}, 5 \mathrm{mM}$ imidazole) on ice and lysed using the OneShot (Constant Systems) at 1.23 kbar. Before cell disruption, $1 \mathrm{mM}$ phenylmethylsulfanyl-fluoride (PMSF) was added. The lysate was then centrifuged at $35,000 \times g$ and $4{ }^{\circ} \mathrm{C}$ for $20 \mathrm{~min}$. The supernatant was loaded into a $2 \mathrm{~mL}$ Ni-Sepharose 6 Fast Flow matrix. After consecutively washing the column with $10 \mathrm{~mL}$ of each of the two wash buffers (20 mM Tris- $\mathrm{HCl}$ (pH 8), $300 \mathrm{mM} \mathrm{NaCl}, 37.5 \mathrm{mM}$ imidazole (Wash Buffer 1) or $50 \mathrm{mM}$ imidazole (Wash Buffer 2)), the complex was eluted with $20 \mathrm{~mL}$ elution buffer (20 mM Tris- $\mathrm{HCl}$ (pH 8), $150 \mathrm{mM} \mathrm{NaCl}, 300 \mathrm{mM}$ imidazole) in $2 \mathrm{~mL}$ fractions. The elution fractions containing the target protein were further purified by ion-exchange chromatography performed with a Resource Q $1 \mathrm{~mL}$ column connected to an ÄKTA purifier 10 system. The sample was loaded onto the column that was equilibrated with Buffer A (20 mM Tris- $\mathrm{HCl}(\mathrm{pH} 8))$ and eluted with a linear gradient against Buffer $\mathrm{B}(20 \mathrm{mM}$ Tris- $\mathrm{HCl}(\mathrm{pH} 8), 1 \mathrm{M} \mathrm{NaCl})$. Relevant fractions were combined and concentrated using Sartorius Vivaspin centrifugal concentrators with a molecular weight cut-off of $10 \mathrm{kDa}$. This sample was loaded onto a Superdex 75 10/300 GL column connected to an ÄKTA purifier 10 system, and purified in a gel filtration buffer (10 mM Tris- $\mathrm{HCl}(\mathrm{pH} 8), 150 \mathrm{mM} \mathrm{NaCl})$.

\subsection{Size-Exclusion Chromatography-Multiple-Angle Light Scattering (SEC-MALS)}

Protein samples were injected into a Superdex 200 increase 10/300 GL column, equilibrated with a gel filtration buffer (10 mM Tris- $\mathrm{HCl}(\mathrm{pH} 8), 150 \mathrm{mM} \mathrm{NaCl})$. The SEC column was linked to a static 3-angle light-scattering detector (miniDAWN Treos II) and a refractive-index detector (Optilab T-rEX) (Wyatt Technology, Santa Barbara, CA, USA). Data were collected every second at a flow rate of $0.5 \mathrm{~mL} / \mathrm{min}$. Data analysis was carried out using ASTRA VII software, yielding the molar mass for each fraction. In addition, protein-conjugate analysis was performed to calculate the molar mass of each component of a protein complex. The light-scattering detectors were normalised, and data quality was assessed by a BSA standard (Thermo, Waltham, MA, USA).

\subsection{PpiD Purification}

E. coli PpiD was expressed from plasmid pASK-IBA3C encoding PipD-Strep (kindly provided by HG Koch, Freiburg) in BL21 (DE3) cells in LB medium containing $25 \mu \mathrm{g} / \mathrm{mL}$ chloramphenicol at $37{ }^{\circ} \mathrm{C}$ until the culture had reached an $\mathrm{OD}_{600}$ of 0.6 . The culture was induced by the addition of anhydrotetracycline to a final concentration of $200 \mu \mathrm{g} / \mathrm{L}$ for $2 \mathrm{~h}$ at $37^{\circ} \mathrm{C}$. Cells were harvested by centrifugation at $10,000 \times g$ and $4{ }^{\circ} \mathrm{C}$ for $15 \mathrm{~min}$. Cell pellets were resuspended in Buffer A (50 mM Tris- $\mathrm{HCl}$ (pH 7.4), $50 \mathrm{mM} \mathrm{NaCl}, 1 \mathrm{mM}$ Ethylen-diamino-tetra-acidic acid (EDTA)) on ice and lysed using the OneShot instrument at 1.23 kbar. Before cell disruption, $1 \mathrm{mM}$ PMSF was added. The lysate was then centrifuged at $35,000 \times g$ and $4{ }^{\circ} \mathrm{C}$ for $45 \mathrm{~min}$. The pellet was resuspended in Buffer A containing 
$1 \% \mathrm{~N}$-lauroylsarcosine to solubilise the inner membrane at $4{ }^{\circ} \mathrm{C}$ overnight. The solubilised membrane fraction was collected by ultracentrifugation at $110,000 \times \mathrm{g}$ at $4{ }^{\circ} \mathrm{C}$ for $40 \mathrm{~min}$ and loaded onto $1 \mathrm{~mL}$ of Strep-Tactin XT matrix. After washing the matrix with $5 \mathrm{~mL}$ of Buffer $\mathrm{W}(100 \mathrm{mM}$ Tris- $\mathrm{HCl}(\mathrm{pH} 8)$, $150 \mathrm{mM} \mathrm{NaCl}, 1 \mathrm{mM}$ EDTA), the protein was eluted with $3 \mathrm{~mL}$ of Buffer BXT (100 mM Tris- $\mathrm{HCl}$ (pH 8), $150 \mathrm{mM} \mathrm{NaCl}, 1 \mathrm{mM}$ EDTA, $50 \mathrm{mM}$ biotin) in $500 \mu \mathrm{L}$ fractions. The elution fractions containing the target protein were further purified by gel filtration chromatography performed with a Superdex 200 increase 10/300 GL column connected to an ÄKTA purifier 10 system in Buffer A.

\subsection{Spheroplast Preparation}

For cross-linking and binding assays, spheroplasts were generated after centrifugation of $E$. coli BL21 (DE3) harbouring plasmid pMAL-p5X (NEB) at 5000 $\times \mathrm{g}$ and $4{ }^{\circ} \mathrm{C}$ for $15 \mathrm{~min}$. The cell pellet was resuspended in an ice-cold spheroplast buffer ( $20 \mathrm{mM}$ Tris- $\mathrm{HCl}$ ( $\mathrm{pH} 8), 0.5 \mathrm{M}$ sucrose) and lysozyme $(20 \mu \mathrm{g} / \mathrm{mL})$, and incubated for $5 \mathrm{~min}$ at room temperature (RT). Then, $1.6 \mathrm{mM}$ EDTA was added, and the sample was incubated for $7 \mathrm{~min}$ at RT [16]. Finally, $10 \mathrm{mM} \mathrm{MgCl}_{2}$ was added to the spheroplasts for stabilization.

\subsection{Liposomes and Proteoliposomes}

E. coli lipids (Avanti) 1-palmitoyl-2-oleoyl-sn-glycero-3-phospho-ethanolamine (POPE) and 1-palmitoyl-2-oleoyl-sn-glycero-3-phosphoglycerol (POPG) were dissolved in dichloromethane and evaporated under a stream of nitrogen. The dry lipid film was dissolved in Buffer A $(50 \mathrm{mM}$ Tris- $\mathrm{HCl}$ (pH 7.4), $50 \mathrm{mM} \mathrm{NaCl}, 1 \mathrm{mM}$ EDTA). A 7:3 mixture of PE and PG was used, mimicking the membrane-lipid composition of E. coli. Liposomes with an average diameter of $250 \mathrm{~nm}$ were generated by extrusion (Mini extruder, Avanti Polar Lipids. Inc.) 31 times through a $0.4 \mu \mathrm{m}$ polycarbonate membrane (Whatman). For preparing the proteoliposomes, purified PpiD protein was mixed with a lipid/protein ratio of at least 10,000:1 ( $\mathrm{mol} / \mathrm{mol})$ and extruded as described above.

\section{Results}

\subsection{Expression and Purification of Central Spike Complex}

The T4 central spike complex was purified from E. coli BL21 harbouring plasmid pEEva2, encoding a C-terminal fragment of the gp5C $\beta$-helix comprising amino acid residues 484-575 and gp5.4 (Figures 1 and 2A). Gp5C $\beta$ contains an N-terminal 6xHis-tag followed by a TEV cleavage site. Full-length C-terminal $\beta$ helix (352-575) is a natural cleavage product of gp5 that appears during assembly of the T4 phage particle [17]. The central spike complex was expressed from cells that were grown overnight at $18{ }^{\circ} \mathrm{C}$ in a shaking incubator. The protein complex was affinity-purified by Ni-NTA, followed by ion-exchange chromatography and gel filtration, resulting in a fraction that mainly consisted of the trimeric complex of gp5C $\beta$ with a bound gp5.4 monomer giving an apparent molecular weight (MW) of the complex of about $38 \mathrm{kDa}$ in SDS-PAGE (Figure 2B). Most of the complex was therefore stable in SDS, and only a fraction was dissociated into separate components: a trimeric gp5C $\beta(3 \times 11.9 \mathrm{kDa})$ and a gp5.4 monomer of about $11 \mathrm{kDa}$ with apparent MWs of 26 and $12 \mathrm{kDa}$, respectively. 
A

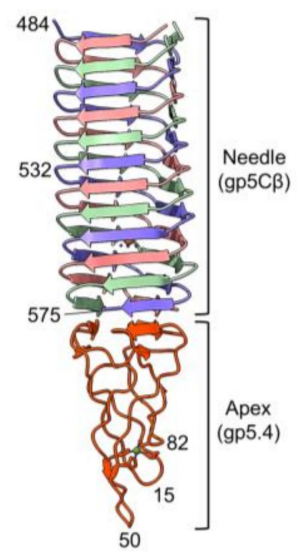

B

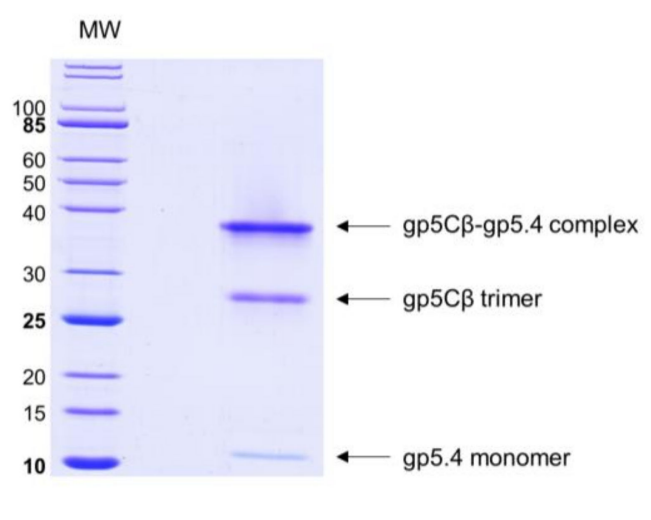

Figure 2. (A) Fragment of central-spike-complex structure comprising the amino acid residues 484-575 of gene 5 with the entire gp5.4. Each chain is a distinct colour. Residue numbers given for strategic positions. (B) Purification of central spike complex. Coexpression of the amino acid residues 484-575 of gp5 and gp5.4 resulted in the gp5C $\beta$-gp 5.4 complex (apparent $\mathrm{MW}=38 \mathrm{kDa}$ ). The complex partially dissociated after SDS-PAGE into a $26 \mathrm{kDa}$ gp $5 \mathrm{C} \beta$ trimeric helix and a $12 \mathrm{kDa}$ monomeric 5.4 apex.

The stoichiometry of the complex was analysed by size-exclusion chromatography and multiangle light scattering (SEC-MALS). The purified gp5C $\beta-5.4$ complex was eluted in a single peak with a retention time of $29.16 \mathrm{~min}$ (Figure 3A). Total molecular weight was determined to be $45.4 \mathrm{kDa}$ (Figure 3B).

A

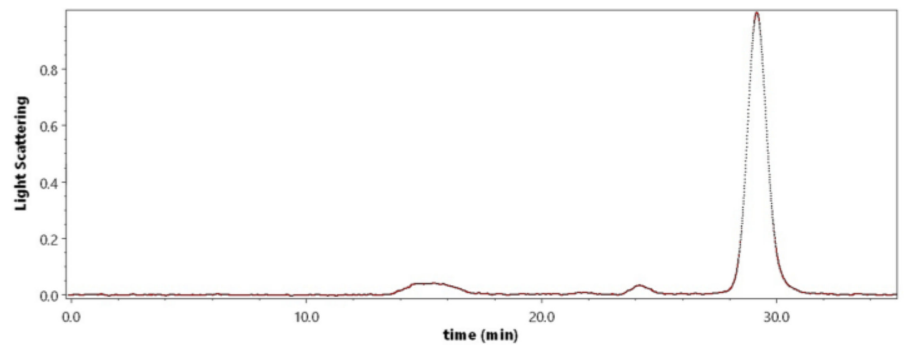

B

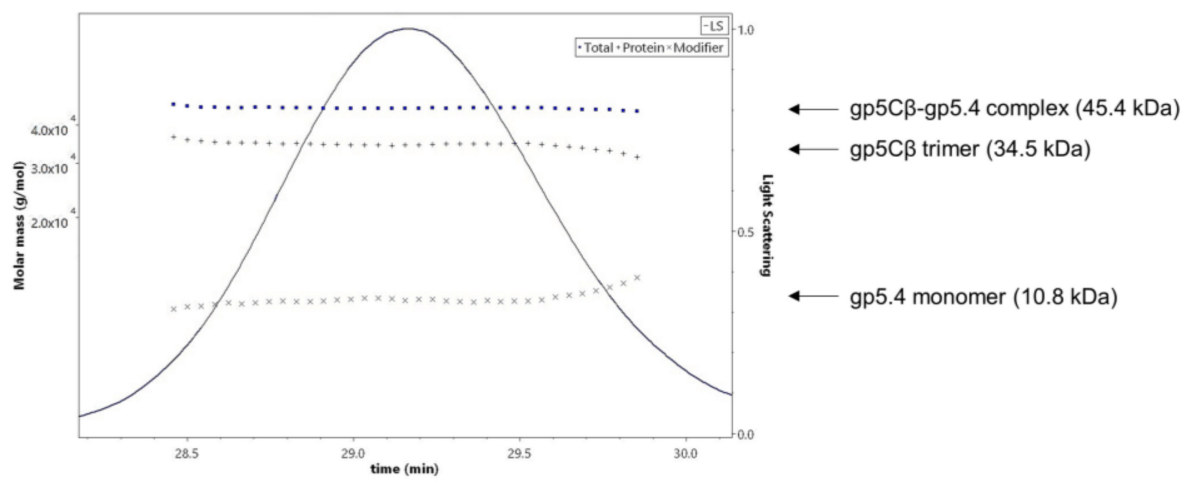

Figure 3. (A) Size-exclusion multiangle-light-scattering (SEC-MALS) analysis of gp5C $\beta$-gp5.4 complex. Affinity-purified complex separated on a Superdex 200 increase where it was eluted at a retention time of $29.16 \mathrm{~min}$. (B) Fractions containing the complex were analysed with a $90^{\circ}$ light-scattering detector verifying its composition and the stoichiometry of its components. The complex was composed of 3 molecules of the $\beta$ helix and one molecule of the gp5.4 protein. Calculated molecular weights (dotted lines, left $Y$ axis) were consistent with theoretical molecular weights. 
In addition, protein-conjugate analysis was performed to calculate the molar mass of each component of the conjugate complex. The molar mass of gp5C $\beta$ and gp5.4 was determined to be 34.5 and $10.8 \mathrm{kDa}$, respectively. These fit with the calculated MW of the complex of $46 \mathrm{kDa}$, consisting of a triple gp5C $\beta$ helix and one copy of gp5.4. Following the molecular weight across the entire peak showed homogeneous distribution both of the complex and of each component. Treatment of the complex with 15\% tri-chloro-acetic acid (TCA) resulted in the dissociation of gp5.4 from gp5C $\beta$, but leaving the triple helix intact. SDS gel electrophoresis revealed two bands with apparent molecular weights of about 26 and $12 \mathrm{kDa}$, respectively (Figure 2B). Taken together, MALS analysis corroborated an exact 3:1 molecular ratio between gp $5 \mathrm{C} \beta$-helix and gp5.4. It also demonstrated that the complex was homogeneous and stable in solution.

\subsection{The gp5Cß-gp5.4 Complex Does Not Bind to Liposomes and Weakly to Spheroplasts}

Since it is plausible that the central spike complex is involved in contacting or perforating the inner membrane, the purified gp5C $\beta$-gp5.4 complex was tested for its binding to liposomes and spheroplasts. The liposomes were generated from 70\% 1-palmitoyl-2-oleoyl-phospho-ethanolamine (POPE) and $30 \%$ 1-palmitoyl-2-oleoyl-phospho-glycerol (POPG) with an extruder to obtain an average diameter of $250 \mathrm{~nm}$ of unilamellar liposomes. The purified gp5C $\beta$-gp5.4 complex was added to the liposomes at room temperature for $30 \mathrm{~min}$ and then applied to an airfuge at $20 \mathrm{psi}$ for $10 \mathrm{~min}$. The supernatant and liposomes in the pellet were then analysed by SDS-PAGE (Figure 4A). The gp5C $\beta$-gp5.4 complex and gp27 control remained in the supernatant, whereas the SecA protein used as an additional control showed binding to the liposomes.

A

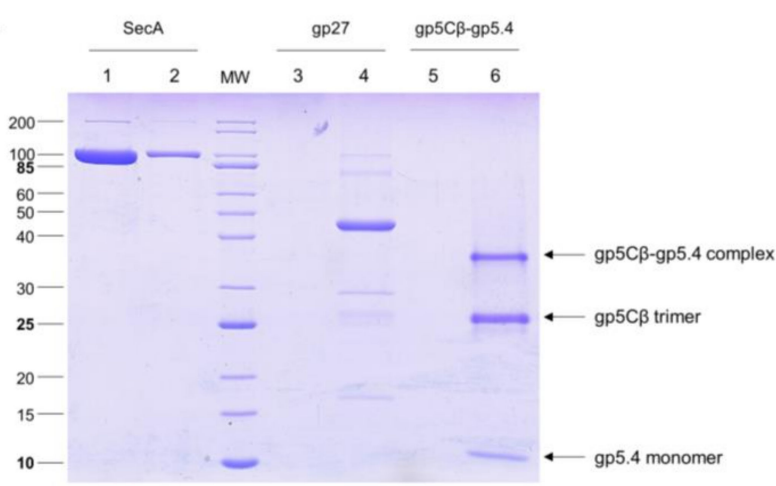

B

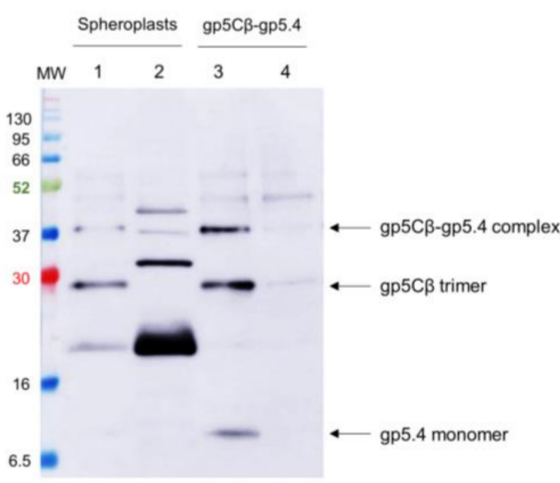

Figure 4. (A) The gp5C $\beta$-gp5.4 complex does not bind to liposomes. The purified complex was incubated at room temperature for $30 \mathrm{~min}$ to preformed liposomes (70\% 1-palmitoyl-2-oleoylphospho-ethanolamine (POPE), 30\% 1-palmitoyl-2-oleoyl-phospho-glycerol (POPG)) and separated after centrifugation (Lane 5 pellet, Lane 6 supernatant). As controls, purified SecA protein (Lane 1 pellet, Lane 2 supernatant) and purified T4 gp27 (Lane 3 pellet, Lane 4 supernatant) were added to the liposomes and centrifuged. SecA was found in the pellet (Lane 1), and the gp5C $\beta$-gp5.4 complex (Lane 6) and gp27 (Lane 4) were found in the supernatant. (B) The gp5C $\beta$-gp5.4 complex binds weakly to spheroplasts. Incubation of the complex for $20 \mathrm{~min}$ with spheroplasts was followed by centrifugation, SDS PAGE, and a Western blot with antiserum to gp5.4 (Lanes 1 and 2). A portion of the complex was found in the pellet fraction (Lane 2), whereas the control without spheroplasts mostly showed the gp5C $\beta$-gp5.4 complex in the supernatant (Lane 3, Lane 4 pellet).

Spheroplasts were prepared from E. coli BL21 by osmotic-shock treatment with lysozyme and EDTA for $10 \mathrm{~min}$. $20 \mathrm{mM} \mathrm{MgCl} 2$ was added to the cell suspension to stabilise the spheroplasts. Then, $25 \mu \mathrm{g}$ of the purified gp5C $\beta-5.4$ complex was added to the spheroplasts and incubated at room temperature for $20 \mathrm{~min}$, followed by centrifugation at $5000 \times \mathrm{g}$. The supernatant and spheroplast pellet were analysed by cross-linking and Western blotting (Figure 4B). Only small amounts of the purified complex were found in association with the spheroplasts. 


\subsection{Interaction of gp5C $\beta-g p 5.4$ Complex with PpiD in the Periplasm}

Most likely, the central spike complex remains in the periplasm after penetration through the outer membrane [2]. To investigate whether it transiently interacts with a periplasmic component on the periplasmic surface of the inner membrane, spheroplasts were prepared, followed by cross-linking treatment (Figure 5). The purified gp5C $\beta$-gp5.4 complex (Lane 2) was incubated with the spheroplasts for $10 \mathrm{~min}$ in the presence of $0.5 \%$ formaldehyde at room temperature, purified by affinity chromatography, and analysed by SDS-PAGE (Lanes 1 and 3). For the control, an aliquot of the formaldehyde-treated sample was heated to $100{ }^{\circ} \mathrm{C}$ for $10 \mathrm{~min}$ (Lane 3), which resulted in a separation of the cross-links. The formaldehyde treatment resulted in an additional band at about $75 \mathrm{kDa}$ (indicated by an asterisk in Lane 1) that was further processed for matrix-assisted laser-desorption/-ionization (MALDI) analysis [18]. This analysis revealed gp5C and the PpiD protein as cross-link products with a probability of over $95 \%$.

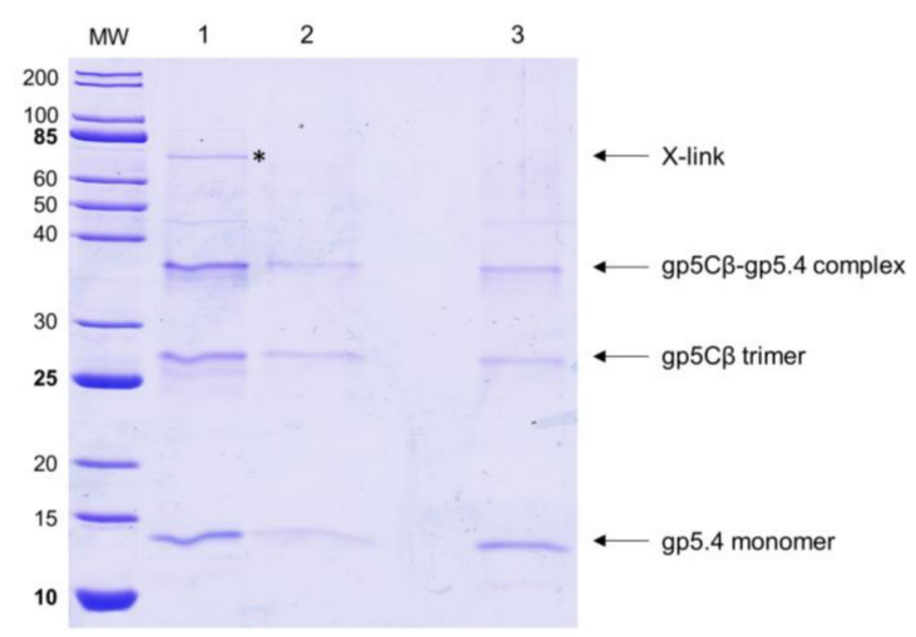

Figure 5. The gp5C $\beta$-gp5.4 complex interacts with spheroplast PpiD. The purified complex was incubated with spheroplasts in the presence of $0.5 \%$ formaldehyde for $10 \mathrm{~min}$ (Lanes 1 and 3). After affinity purification, the complex coeluted with periplasmic protein PpiD, which was identified by matrix-assisted laser-desorption/-ionization (MALDI) analysis (Lane 1, asterisk). As control, the affinity-purified gp5C $\beta$-gp5.4 complex is shown (Lane 2). To break the cross-link, sample was heated at $100{ }^{\circ} \mathrm{C}$ (lane 3$)$.

To verify such an interaction, PpiD was purified and added to the liposomes in a reconstitution setup. The resulting proteoliposomes containing PpiD were then tested for binding the gp $5 \mathrm{C} \beta$-gp5.4 complex (Figure 6A). The PpiD proteoliposomes were incubated with the purified complex at room temperature for $10 \mathrm{~min}$ and sedimented by centrifugation. The proteoliposomes were indeed capable to reproducibly bind a small portion of the complex (Lane 3), in contrast to empty liposomes (Lane 5). This result is consistent with the observation of the cross-linking experiment, and suggests that PpiD might play a supporting role for the infection process of T4. To test this idea, the plating efficiency of T4 was analysed with the ppiD deletion strain JW0431 $\Delta p p i D$ and compared to the parental BW25113 (Figure $6 \mathrm{~B}$ ). The plating efficiency of T4 on JW0431 $p p p i D$ was reduced to $78 \% \pm 2 \%$ (Figure $6 \mathrm{~B}$ ). When the plasmid-encoded PpiD was expressed in JW0431 $\Delta p p i D$, plating efficiency was restored. 


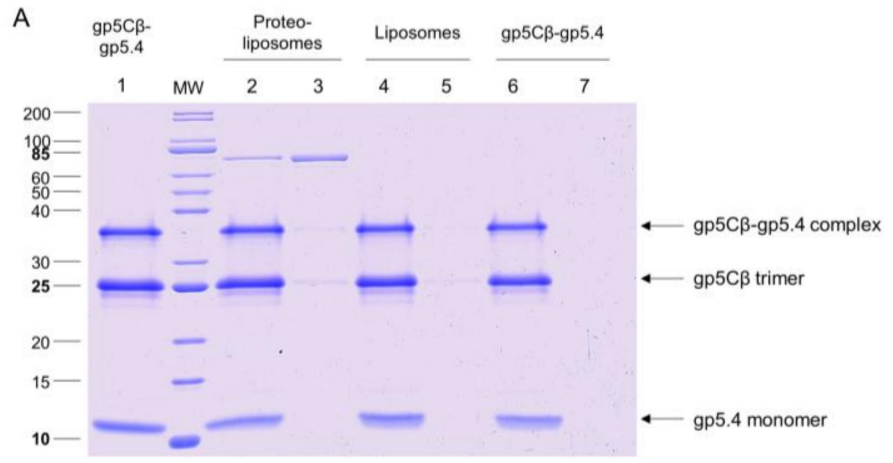

B

Figure 6. (A) Binding of gp5C $\beta$-gp5.4 complex to PpiD proteoliposomes. Purified PpiD protein was reconstituted into proteoliposomes (70\% POPE, 30\% POPG). Purified complex (Lane 1) was added and incubated at room temperature (RT) for $10 \mathrm{~min}$. After spinning down proteoliposomes, the complex copelleted with the PpiD proteoliposomes (Lane 2 supernatant, Lane 3 pellet), but it did not bind to liposomes (Lane 4 supernatant, Lane 5 pellet) nor was it present as aggregate (Lane 6 supernatant, Lane 7 pellet). (B) Efficiency of plating (EOP) is affected by deletion of the ppiD gene. JW0431 cells, deficient for expressing PpiD and its parent strain BW25113, were tested for their efficiency to form plaques after T4 infection. Number of plaques was reduced to $78 \% \pm 2 \%$, an indication that T4 phage infection is less efficient when PpiD is missing. Plasmid-encoded PpiD in the JW0431 deletion strain restored EOP to $100 \%$.

\subsection{The Fate of the Central Spike Complex after the Infection Process}

Several gp5 and gp5.4 peptide-related antisera were raised and tested on purified phage preparations. A serum recognising gp5C in the phage was directed against the C-terminal 20 residues (537-556) of gp5C with antigenic peptide KVAGTVDWDVGGDWTEKMAS. This serum allowed for recognising the gp5C-containing central spike complex in the phage particle (Figure 7A), and to follow the fate of the protein after infection of E. coli BL21 bearing plasmid pMAL-p5X, expressing MalE (Figure 7B,C). Exponentially growing cells were infected with a multiplicity of infection (MOI) of 20, and samples were taken at 2, 5, and 10 min postinfection (pI). Samples were spun down to remove the nonadsorbed phage, and infected cells present in the pellet were converted to spheroplasts. The released periplasmic shock fluids (odd-numbered lanes) were separated from the spheroplasts (even-numbered lanes). Samples were separated by PAGE and analysed by Western blot. Results showed that the gp5C-containing complex was present in the pellet fraction (Lanes 4, 6, and 8), and also progressively appeared in the periplasmic fluid (Lanes 3, 5, and 7). The complex remained stable at least until $10 \mathrm{~min}$ pI (Lanes 7 and 8).

A

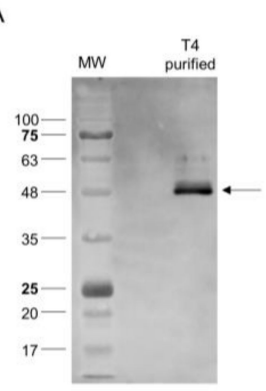

B

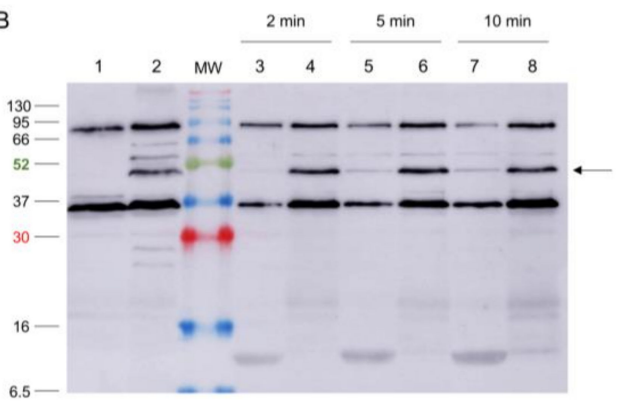

C

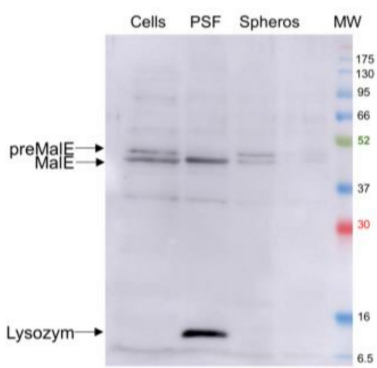

Figure 7. (A) Presence of gp5C in CsCl-purified T4 phage visualised by Western blot. (B) Fate of gp5C (bands marked with an arrow) from infecting phage at 2 (Lanes 3 and 4), 5 (Lanes 5 and 6) and 10 min post infection (pI; Lanes 7 and 8). Gp5C found in spheroplasts (Lanes 4, 6, and 8) and periplasmic fluid (Lanes 3, 5, and 7). For control, uninfected (Lane 1) and whole T4-infected cells (10 min pI; Lane 2) were analysed. (C) Fractionation of pre-MalE and MalE in periplasmic shock fluid (PSF) and in spheroplasts (Spheros) as controls. 


\section{Discussion}

The infection process of myophage T4 involves an astounding cascade of conformational events, and this is exemplary in protein biochemistry. Remarkably, the self-triggered multistep process is independent of any outside energy input, similar to the mechanism of an old-fashioned time watch driven to operate for days by a key-loaded spring. The first steps of the phage adsorption and interaction of tail fibres with the receptors of the host cell [3], the movement of the short fibres, and the contraction of the tail sheath $[4,5]$ are quite well-understood, whereas the molecular events that occur when the phage is penetrating the outer and inner host membrane are still unknown.

When the tail tube, driven by sheath contraction, moves through the baseplate, it takes with itself the central hub proteins of the baseplate, gp5, gp5.4, and gp27, together with gp48 and gp54. Therefore, the structure that enters the host periplasm is a capped tail tube structure, as depicted in Figure 1. In the present study, we followed the fate of the gp5 and gp5.4 proteins at the leading end of the periplasm-entering tail tube. In the phage particle, gp5 is a cleaved protein that is present as two distinct fragments, gp5* and gp5C [17]. Gp5C is folded into a triple helix that tightly binds 5.4 and withholds its triple-helical structure [9], even after denaturing treatments on SDS-PAGE giving rise to an apparent $48 \mathrm{kDa}$ protein band (Figure $7 \mathrm{~A}$ ). The stability of the complex allowed for purifying a fragment of gp5C (484-575), termed gp5C $\beta$, with gp5.4 from E. coli cells encoded on a plasmid [10]. Expressed from the E. coli cells, the two proteins formed a complex, gp5C $\beta$-gp5.4, which was partially stable on SDS-PAGE (Figure 2B), and encompassed 3 copies of gp5C $\beta$ and one copy of gp5.4, as was verified by MALS analysis (Figure 3).

The purified complex was then used for binding experiments in vitro to uncover possible interacting partner proteins. First, the addition to spheroplasts and liposomes did not show strong interaction (Figure 4), suggesting that the central spike complex freely diffuses in the periplasm, as was suggested earlier [19]. However, when we used formaldehyde as a chemical cross-linker to stabilise possible interactions with spheroplasts, we found that PpiD copurified with the gp5C $\beta-5.4$ complex (Figure 5). This interaction was verified with proteoliposomes that were reconstituted with purified PpiD (Figure 6A). PpiD proteoliposomes showed enhanced binding of the added central spike complex when compared with that of pure liposomes. PpiD is a periplasmic chaperone, anchored to the inner membrane that is devoted to binding and stabilising proteins. Regarding its function for T4 infection, it could either catalyse the interaction of the tail tube with the inner membrane for penetration or simply bind the already dissociated gp5C $\beta$-gp5.4 complex to prevent an inhibitory role for efficient tail tube-inner membrane interaction. Additional experiments are required to clarify these points.

The involvement of PpiD in the T4 infection and propagation process was then studied with efficiency of plating (EOP). This assay quantified the proportion of infecting phage that successfully led to the formation of a plaque. When we compared the parental strain with the deletion mutant of PpiD, a reduction in EOP to less than $80 \%$ was determined. This points to a nonessential but supporting role of PpiD in the T4 infection process. Alternatively, other periplasmic chaperones such as SurA or Skp [20] might step in when ppiD is deleted and compensate for the loss of PpiD.

When we followed the gp5C-containing complex in phage particles, the major portion was found in a $48 \mathrm{kDa}$ protein band (Figure 7A,B). Minor bands migrated at 65 and $52 \mathrm{kDa}$, respectively. The known cleavage site at amino acid residue 351 generates a triple helix of gp5C with a theoretical molecular weight of $59 \mathrm{kDa}$. Previously, it was shown that gp5C runs with a lower apparent MW [11], which might be caused by protein folding of the triple helix. After infection, the $48 \mathrm{kDa}$ protein complex remained mainly with the spheroplasts (Figure 7B). Therefore, the central spike complex might indeed come into contact with the cytoplasmic membrane. However, in our analysis with the purified gp5C $\beta$-gp5.4 complex, we did not find a strong binding to spheroplasts. It is, therefore, possible that additional factors are required to support the binding of the T4 tail tube to the membrane surface.

Taken together, our results suggest that the T4 central spike complex enters the periplasm and interacts with low affinity to PpiD. In our analysis, we did not observe stable interaction with the inner membrane, which is required for the phage to establish a DNA-translocating complex. 
Author Contributions: P.G.L., A.K., and D.K. designed the experiments; S.W. and M.M.S. performed the experiments; A.K. and D.K. wrote the paper. All authors have read and agreed to the published version of the manuscript.

Funding: This work was supported by DFG grant KU 749/7-1 (to AK and DK).

Conflicts of Interest: The authors declare no conflict of interest. The funding sponsors had no role in the design of the study; in the collection, analyses, or interpretation of data; in the writing of the manuscript, and the decision to publish the results.

\section{References}

1. Kellenberger, E.; Stauffer, E.; Häner, M.; Lustig, A.; Karamata, D. Mechanism of the long tail-fiber deployment of bacteriophages T-even and its role in adsorption, infection and sedimentation. Biophys. Chem. 1996, 59, 41-59. [CrossRef]

2. Hu, B.; Margolin, W.; Molineux, I.; Liu, J. Structural remodelling of bacteriophage T4 and host membranes during infection initiation. Proc. Natl. Acad. Sci. USA 2015, 112, E4919-E4928. [CrossRef] [PubMed]

3. Islam, M.Z.; Fokine, A.; Mahalingam, M.; Zhang, Z.; Garcia-Doval, C.; van Raaij, M.J.; Rossmann, M.G.; Rao, V.B. Molecular anatomy of the receptor binding module of a bacteriophage long tail fiber. PLOS Pathog. 2019, 15, e1008193. [CrossRef] [PubMed]

4. Leiman, P.; Chipman, P.R.; Kostyuchenko, V.A.; Mesyanzhinov, V.V.; Rossmann, M.G. Three-dimensional rearrangement of proteins in the tail of bacteriophage T4 on infection of its host. Cell 2004, 118, 419-429. [CrossRef] [PubMed]

5. Taylor, N.M.I.; Prokhorov, N.S.; Guerrero-Ferreia, R.; Shneider, M.M.; Browning, C.; Goldie, K.N.; Stahlberg, H.; Leiman, P.G. Structure of the T4 baseplate and its function in triggering sheath contraction. Nature 2016, 533, 346-352. [CrossRef] [PubMed]

6. Kostyuchenko, V.A.; Chipman, P.R.; Leiman, P.G.; Arisaka, F.; Mesyanzhinov, V.V.; Rossmann, M.G. The tail structure of bacteriophage T4 and its mechanism of contraction. Nat. Struct. Mol. Biol. 2005, 12, 810-813. [CrossRef] [PubMed]

7. Moody, M.F. Sheath of bacteriophage T4 3. Contraction mechanism deduced from partially contracted sheaths. J. Mol. Biol. 1973, 80, 613-635. [CrossRef]

8. Leiman, P.G.; Arisaka, F.; van Raaij, M.J.; Kostyuchenko, V.A.; Aksyuk, A.A.; Kanamaru, S.; Rossmann, M.G. Morphogenesis of the T4 tail and tail fibers. Virol. J. 2010, 7, 355. [CrossRef] [PubMed]

9. Arisaka, F.; Kanamaru, S.; Leiman, P.; Rossmann, M. The tail complex of bacteriophage T4. Int. J. Biochem. Cell Biol. 2003, 35, 16-21. [CrossRef]

10. Shneider, M.M.; Buth, S.A.; Ho, B.T.; Basler, M.; Mekalanos, M.M.; Leiman, P.G. PAAR-repeat proteins sharpen and diversify the type VI secretion system spike. Nature 2013, 500, 350-353. [CrossRef] [PubMed]

11. Kanamaru, S.; Gassner, N.C.; Ye, N.; Takeda, S.; Arisaka, F. The C-terminal fragment of the precursor tail lysozyme of bacteriophage T4 stays as a structural component of the baseplate after cleavage. J. Bacteriol. 1999, 181, 2739-2744. [CrossRef] [PubMed]

12. Matern, Y.; Barion, B.; Behrens-Kneip, S. PpiD is a player in the network of periplasmic chaperones in Escherichia coli. BMC Microbiol. 2010, 10, 251. [CrossRef] [PubMed]

13. Antonoaea, R.; Fürst, M.; Nishiyama, K.-I.; Müller, M. The periplasmic chaperone PpiD interacts with secretory proteins exiting from the SecYEG translocon. Biochemistry 2008, 47, 5649-5656. [CrossRef] [PubMed]

14. Manaiatis, T.; Fritsch, E.F.; Sambrook, J. Molecular Cloning: A Laboratory Manual; Cold Spring Harbor Press: New York, NY, USA, 1982.

15. Studier, F.W.; Moffat, B.A. Use of bacteriophage T7 RNA polymerase to direct selective high-level expression of cloned genes. J. Mol. Biol. 1986, 189, 113-130. [CrossRef]

16. Pross, E.; Soussoula, L.; Seitl, I.; Lupo, D.; Kuhn, A. Membrane targeting and insertion of the C-tail protein SciP. J. Mol. Biol. 2016, 428, 4218-4227. [CrossRef] [PubMed]

17. Mosig, G.; Lin, G.W.; Franklin, J.; Fan, W.H. Functional relationships and structural determination of two bacteriophage T4 lysozymes: A soluble (gene e) and a baseplate associated (gene 5) protein. New Biol. 1989, 1, 171-179. [PubMed]

18. Webster, J.; Oxley, D. Protein identification by MALDI-TOF mass spectrometry. Meth. Mol. Biol. 2011, 800, 227-240. 
19. Nishima, W.; Kanamaru, S.; Arisaka, F.; Kitao, A. Screw motion regulates multiple functions of T4 phage protein gene product 5 during cell puncturing. J. Am. Chem. Soc. 2011, 133, 13571-13576. [CrossRef] [PubMed]

20. Mas, G.; Thoma, J.; Hiller, S. The periplasmic chaperones Skp and SurA. Subcell. Biochem. 2019, 92, 169-186. [PubMed]

(C) 2020 by the authors. Licensee MDPI, Basel, Switzerland. This article is an open access article distributed under the terms and conditions of the Creative Commons Attribution (CC BY) license (http://creativecommons.org/licenses/by/4.0/). 